\title{
Phylogeny and expression profiling of $C A D$ and CAD-like genes in hybrid Populus (P. deltoides $\times P$. nigra): evidence from herbivore damage for subfunctionalization and functional divergence
}

\author{
Abdelali Barakat*1, Agnieszka Bagniewska-Zadworna² ${ }^{2}$ Christopher J Frost ${ }^{1,3}$ and John E Carlson*1
}

\begin{abstract}
Background: Cinnamyl Alcohol Dehydrogenase (CAD) proteins function in lignin biosynthesis and play a critical role in wood development and plant defense against stresses. Previous phylogenetic studies did not include genes from seedless plants and did not reflect the deep evolutionary history of this gene family. We reanalyzed the phylogeny of $C A D$ and $C A D$-like genes using a representative dataset including lycophyte and bryophyte sequences. Many CAD/CADlike genes do not seem to be associated with wood development under normal growth conditions. To gain insight into the functional evolution of CAD/CAD-like genes, we analyzed their expression in Populus plant tissues in response to feeding damage by gypsy moth larvae (Lymantria dispar L.). Expression of CAD/CAD-like genes in Populus tissues (xylem, leaves, and barks) was analyzed in herbivore-treated and non-treated plants by real time quantitative RT-PCR.

Results: CAD family genes were distributed in three classes based on sequence conservation. All the three classes are represented by seedless as well as seed plants, including the class of bona fide lignin pathway genes. The expression of some CAD/CAD-like genes that are not associated with xylem development were induced following herbivore damage in leaves, while other genes were induced in only bark or xylem tissues. Five of the CAD/CAD-like genes, however, showed a shift in expression from one tissue to another between non-treated and herbivore-treated plants. Systemic expression of the CAD/CAD-like genes was generally suppressed.

Conclusions: Our results indicated a correlation between the evolution of the CAD gene family and lignin and that the three classes of genes may have evolved in the ancestor of land plants. Our results also suggest that the CAD/CAD-like genes have evolved a diversity of expression profiles and potentially different functions, but that they are nonetheless co-regulated under stress conditions.
\end{abstract}

\section{Background}

Lignin is a phenolic heteropolymer that plays a central role in plant structure by providing rigidity and hydrophobicity to xylem cell walls [1], which facilitates the conduction of water and minerals throughout the plant body [2]. The lignin synthesis pathway involves many substrates and enzymes, among which the CAD enzyme catalyzes the last step in the synthesis of monolignol precursors. While mutations in a single real $C A D$ gene

*Correspondence: aub14@psu.edu, jec16@psu.edu

The School of Forest Resources, and The Huck Institutes of the Life Sciences, Pennsylvania State University, 324 Forest Resources Building, University Park, PA 16802, USA

Full list of author information is available at the end of the article rarely affect growth of transgenic plants, some natural mutants or double mutants have shown abnormal developmental and structural phenotypes. For instance, Arabidopsis plants with double mutations in the two major $C A D$ genes associated with lignin biosynthesis (AtCAD3 and $A t C A D 4$ ) present prostrate stems linked to weakness of the vasculature, as well as a reduction in the stem size and diameter [3]. The natural brown midrib (bm) mutants in sorghum and maize [4-6] which presented $20 \%$ reduction in lignin content $[7,8]$, showed several phenotypic variations including increased lodging, reduced yield, regrowth, height, variation in the flowering time, and tillering in some environments, compared to wild type counterparts. 
Lignin plays an important role in plant defense against insects and pathogens [9-12]. Lignin functions as a physical impediment, either physically blocking pathogen entry or increasing leaf toughness to make chewing by herbivores more difficult. While lignin synthesis is a central component of most plant growth, the rate of lignin synthesis can be accelerated by herbivory or pathogen attack. The rapid deposition of lignin or lignin-like materials may inhibit further growth and confine the invading pathogen or reduce the fecundity of an herbivore [13-18]. For instance, infection of Populus with the rust fungus, Melampsora larici-populina, led to a strong accumulation of monolignols within two days of infection [19]. Similarly, Norway spruce bark and cambium inoculated with the bark beetle associated fungal pathogen, Ceratocystis polonica, became partially or completely lignified surrounding the inoculation site [20,21]. Lignin functions in plant defense against herbivores by increasing leaf toughness and decreasing leaf nutritional content [22]. Moreover, lignin production can be altered in response to herbivore feeding [23]. Considering the importance of lignin in plant defense and the effect of herbivory on the phenylpropanoid pathway [24-29], it is not surprising that previous studies have shown an increase in the expression of $C A D / C A D$-like genes in plants infected with pests and pathogens [30-32].

In Populus, we reported 15 CAD/CAD-like genes distributed in three main classes [33]. However, our phylogeny in that study [33] did not include sequences from seedless plants and could not draw clearly the complete evolutionary history of this gene family. Among the CAD/CAD-like genes in Populus, only two (PoptrCAD4 and PoptrCAD10) have been shown to be preferentially expressed in xylem under normal growth conditions. In contrast, three Populus genes (PoptrCAD7, PoptrCAD12, and PoptrCAD13) are preferentially expressed in leaves, and one gene is preferentially expressed in both leaves and xylem (PoptrCAD9). The functions of Populus CAD/ CAD-like genes, except PoptrCAD4 and PoptrCAD10, are still unknown. Phylogenetic analysis has shown that all the bona fide lignin pathway $C A D$ genes studied so far belong to Class I [33]. Populus Sinapyl Alcohol Dehydrogenase $(S A D)$ genes [33,34] as well as Arabidopsis AtCAD4, AtCAD6, and AtCAD5 genes, which have been shown to be associated with lignin biosynthesis [35], were distributed in Class II. We do not know whether the distribution of $C A D / C A D$-like genes in three main classes reflects a functional divergence and several questions related to the phylogeny and the function of $C A D / C A D$ like genes remain to be answered. For instance, are Class I genes associated only with xylem development while Class II and Class III genes function in defense against biotic stresses? Do genes from the three classes compensate for each other both under normal growth and stress conditions? Will different $C A D / C A D$-like genes respond similarly to herbivore stress? Will herbivore-mediated expression of the $C A D / C A D$-like gene family members differ between physically damaged tissue and systemic, undamaged tissue?

To address these questions, we re-analyzed the phylogeny of $C A D / C A D$-like genes using a dataset that includes sequences from a moss and a lycophyte. We also used quantitative RT-PCR to analyze the expression of ten $C A D / C A D$-like genes in several tissues (leaves, bark, xylem systemic, and undamaged leaf tissue) from hybrid Populus plants challenged with gypsy moth larvae and unchallenged control plants. These studies permitted us to relate the evolution of lignin and the CAD family, and to get insight into the evolution of gene functionalization within the family.

\section{Results}

\section{Phylogeny of CAD/CAD-like genes}

Phylogenetic analysis was conducted using maximum likelihood with protein sequences from over 40 species belonging to various land plant lineages (see Additional file 1 Table S1). The analysis showed that CAD/CAD-like genes are distributed in three major classes (Fig. 1). The distribution of genes in Class I and III are supported with good bootstrap values (100 and 63 for Class I and Class III respectively), while Class II is not as well supported (bootstrap value $=49$ ). Class I (bona fide lignin gene class) and Class III CAD/CAD-like genes include sequences from angiosperm and moss (Physcomitrella). However, Class III is represented by sequences from angiosperms and moss but not the lycopod Selaginella.

In Class I, II, and III, genes from seedless plants cluster separately from the angiosperms. The distribution of $C A D / C A D$-like genes in the tree shows evidence of several plant-specific or lineage specific duplications. Class III includes few genes from both monocots and eudicots but no gymnosperm sequences. In all three classes, sequences from monocots, eudicots, gymnosperms, and seedless plants cluster separately indicating that events that contributed to the duplication of these genes have occurred after the splits of those lineages from each other.

\section{Expression profiling of CAD genes in herbivore-damaged tissues}

In Populus, the $C A D / C A D$-like gene family is represented by 15 genes [33]. However, only two genes (PoptrCAD4 and PoptrCAD10) are preferentially expressed in xylem, where lignin deposition is required to increase plant tensile strength. Most of the remaining genes are induced in leaves and/or bark [33]. To determine which of the Populus $C A D / C A D$-like genes are associated with response to herbivory, we analyzed the expression of $10 C A D / C A D$ - 


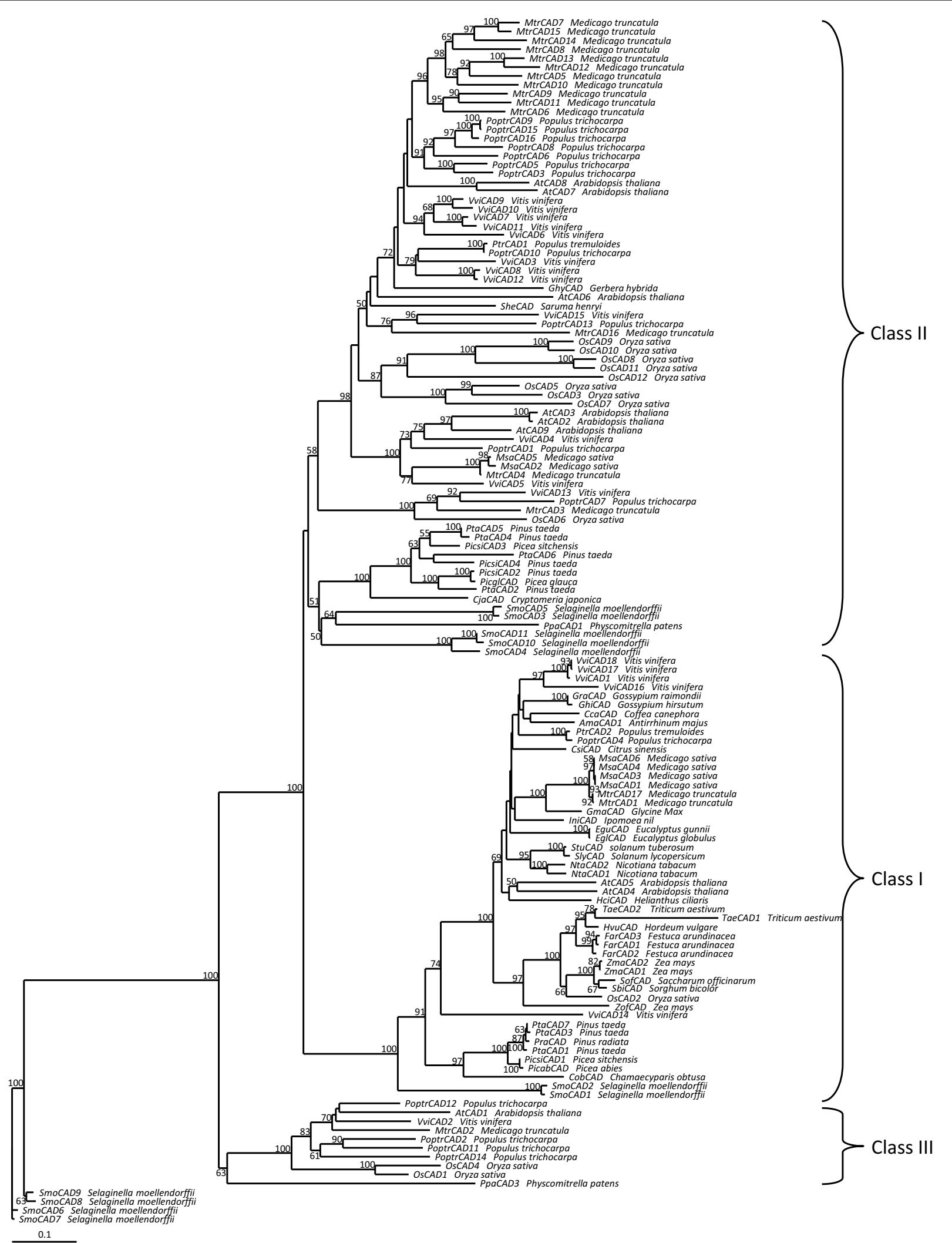

Figure 1 Phylogenetic tree of $C A D / C A D$-like genes. Numbers above branches refer to bootstrap values. Values that were below 50 were not reported. Brackets highlight the three classes of CAD/CAD-like genes. 
like genes using quantitative RT-PCR in various tissues from plants challenged with Lymantria dispar. To address questions related to the evolution of $C A D / C A D$ like genes, we selected ten genes representing all phylogenetic clusters in the three classes of $C A D / C A D$-like genes and presenting different expression patterns in nonstressed plants. We searched for a local response by analyzing the expression in tissues (leaves, bark, and xylem) directly in contact with the herbivores, even though gypsy moth only feed on the foliage. Real time RT-PCR experiments showed that six of the ten CAD/CAD-like genes studied exhibited a preferential expression in at least one of the tissues analyzed (Fig. 2). The expression profiles of $C A D / C A D$-like genes in tissues from stressed plants can be divided in five groups: The group 1 genes (PoptrCAD4 and PoptrCAD11) showed a preferential expression in xylem. The group 2 genes (PoptrCAD4, PoptrCAD3, and PoptrCAD15) showed an induced expression in leaves. The only gene in group 3 (PoptrCAD7) is highly induced in the bark. Group 4 genes (PoptrCAD2, PoptrCAD5, PoptrCAD12, and PoptrCAD14) showed similar expression profiles in all tissues from stressed and control plants, and thus appear to be constitutively expressed. Finally, the expression of the one gene in Group 5 (PoptrCAD10) is suppressed in xylem.

While PoptrCAD4 from group 1 was preferentially expressed in xylem, PoptrCAD11 showed similar expression levels in xylem, bark, and leaves under normal growth conditions [33]. The expression of PoptrCAD11 and PoptrCAD4 at least doubled in xylem tissues challenged with Lymantria dispar. PoptrCAD4 and PoptrCAD10, which are associated with lignin biosynthesis during development, present different expression patterns under herbivore stress. While PoptrCAD4 is highly induced in xylem from stressed plants, PoptrCAD10 showed a lower level of expression in treated plants. PoptrCAD7, which showed a preferential expression in the leaf under normal growth conditions, changed the location (shifted) of its preferential expression to the bark under herbivory conditions. PoptrCAD2, PoptrCAD5, PoptrCAD12 and PoptrCAD14 did not show any expression difference in tissues from stressed and non-stressed plants. One-way ANOVA results showed a large variation in gene expression between tissues in non-treated plants (except genes PoptrCAD2, PoptrCAD11, PoptrCAD12, and PoptrCAD15) and herbivore treated plants (except PoptrCAD12 and PoptrCAD15).

\section{Expression profiling of CAD genes in systemic leaves}

To test if the differential expression of CAD/CAD-like genes shown in Populus plants challenged with herbivores was systemic, we analyzed the expression in leaves that were not in contact with herbivores (systemic leaves)
(Fig. 3). Five of the ten $C A D / C A D$-like genes (PoptrCAD10, PoptrCAD15, PoptrCAD12, PoptrCAD5, and PoptrCAD11) were significantly down-regulated in the systemic leaves. The other five genes, PoptrCAD2, PoptrCAD3, PoptrCAD4, PoptrCAD7 and PoptrCAD14, were not affected by herbivory in systemic leaves. To compare the response of $C A D / C A D$-like genes between directly treated and systemic leaves, we analyzed the expression level of two different genes (PoptrCAD3 and PoptrCAD15). The analysis showed that the expression of PoptrCAD3 and PoptrCAD15 was higher in the directly treated leaves from stressed plants compared to control plants (Fig. 4). However, the expression of these two genes was very low in systemic leaves from stressed plants compared to control plants.

\section{Expression of the rubisco gene in directly treated and systemically induced tissues}

Plants tend to redirect their metabolism in response to herbivory in ways that affect both the damaged and undamaged parts of the plant $[36,37]$. To check whether the low expression level of $C A D / C A D$-like genes observed in non-treated tissues was linked to an overall lower metabolism in those tissues, we analyzed the expression of the rubisco gene, as rubisco and other photosynthetic genes are known to be suppressed by herbivory [38-42]. In our experiment, the expression of the rubisco gene was induced in directly treated leaves and was repressed in the systemic undamaged leaves (Fig. 5).

\section{Discussion}

Phylogenetic analysis of $C A D / C A D$-like genes published recently by our group [33] used a sequence dataset that did not include seedless plant sequences. This limited insights into the evolution of $C A D / C A D$-like genes in relationship with the evolution of lignin in land plants. Here we re-analyzed the phylogeny of CAD/CAD-like genes using sequences from the ten plants for which the genomes are completely sequenced including the moss Physcomitrella and the lycopod Selaginella. Phylogenetic analysis showed that $C A D / C A D$-like genes, except four Selaginella sequences (SmoCAD6, SmoCAD7, SmoCAD8, and SmoCAD9) are distributed in three classes. This resulting tree is in agreement with our previous results [33], however the origin of all classes of $C A D /$ $C A D$-like genes in seed plants from genes previously residing in mosses is now documented. The four Selaginella sequences that do not cluster with the other genes could correspond to other dehydrogenase genes that are highly similar to $C A D / C A D$-like genes. Two of the three classes (Class I and Class III) are well supported while Class II is not. Class I and Class II include genes from both Physcomitrella and Selaginella. This indicates that the evolution of these two classes happened in the ances- 


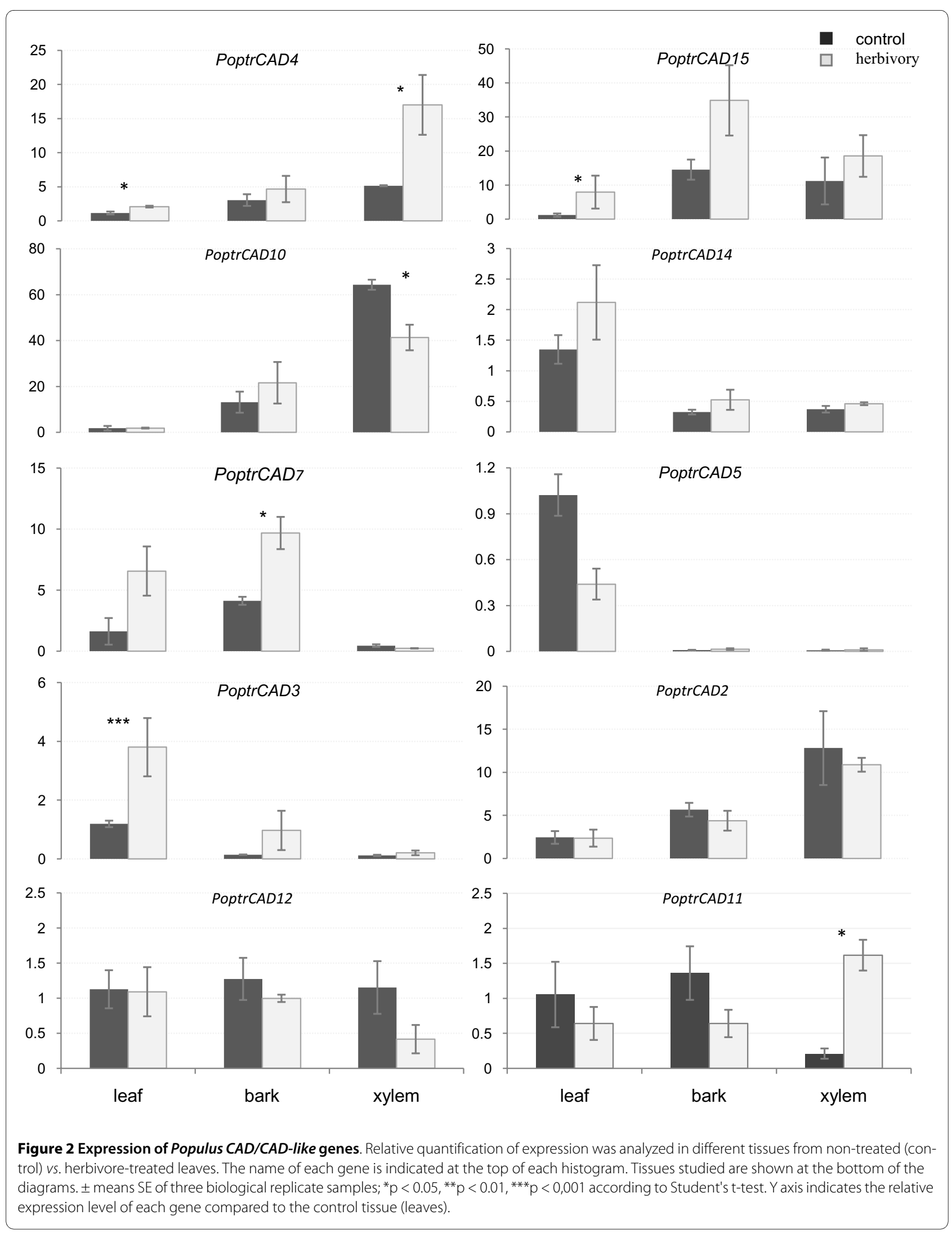




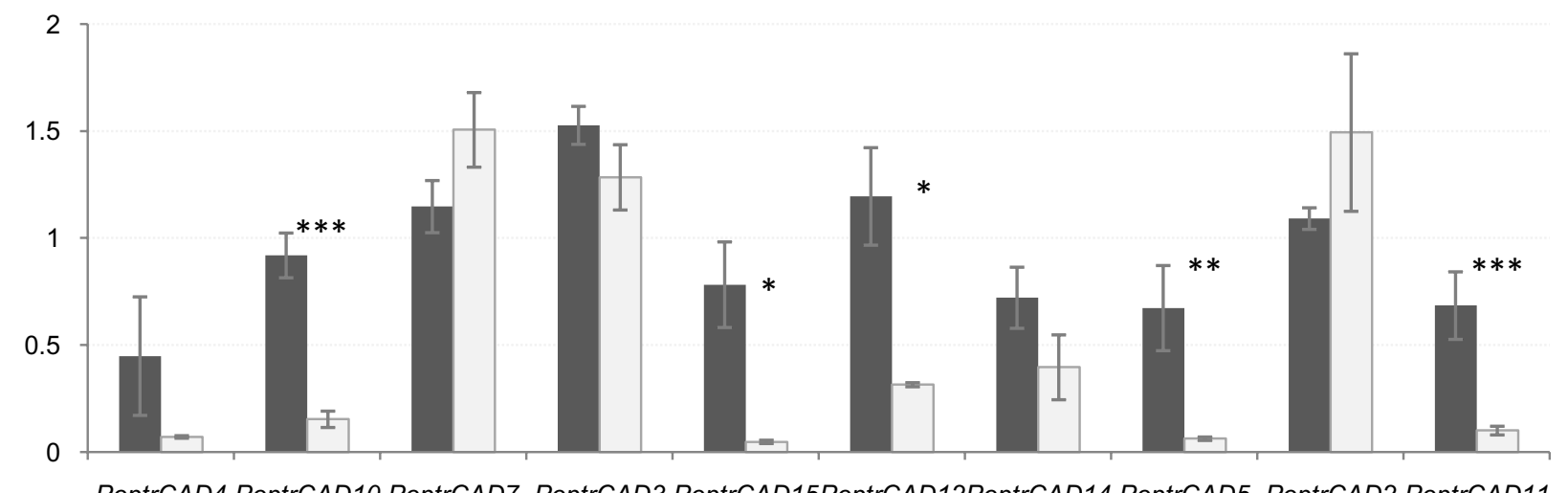

PoptrCAD4 PoptrCAD10 PoptrCAD7 PoptrCAD3 PoptrCAD15PoptrCAD12PoptrCAD14 PoptrCAD5 PoptrCAD2 PoptrCAD11

Figure 3 Relative expression of $C A D / C A D$-like genes in directly treated vs systemically induced leaves from Populus plants. \pm means $S E$ of three biological replicate samples.; ${ }^{*} p<0.05,{ }^{* *} p<0.01$, ${ }^{* * *} p<0,001$ according to Student's t-test. Y axis indicates the relative expression level of each gene compared to the control tissue (non-treated leaves).

tor of land plants. Class III includes a sequence from Physcomitrella which is the most basal among the Class III genes, but neither Selaginella nor gymnosperm sequences. This suggests that Class III may represent the first $C A D$ genes that evolved with or after the divergence of mosses from lycophytes. The lack of gymnosperm sequences in this class either represents gene loss following divergence of Classes I and II, or is perhaps an artifact

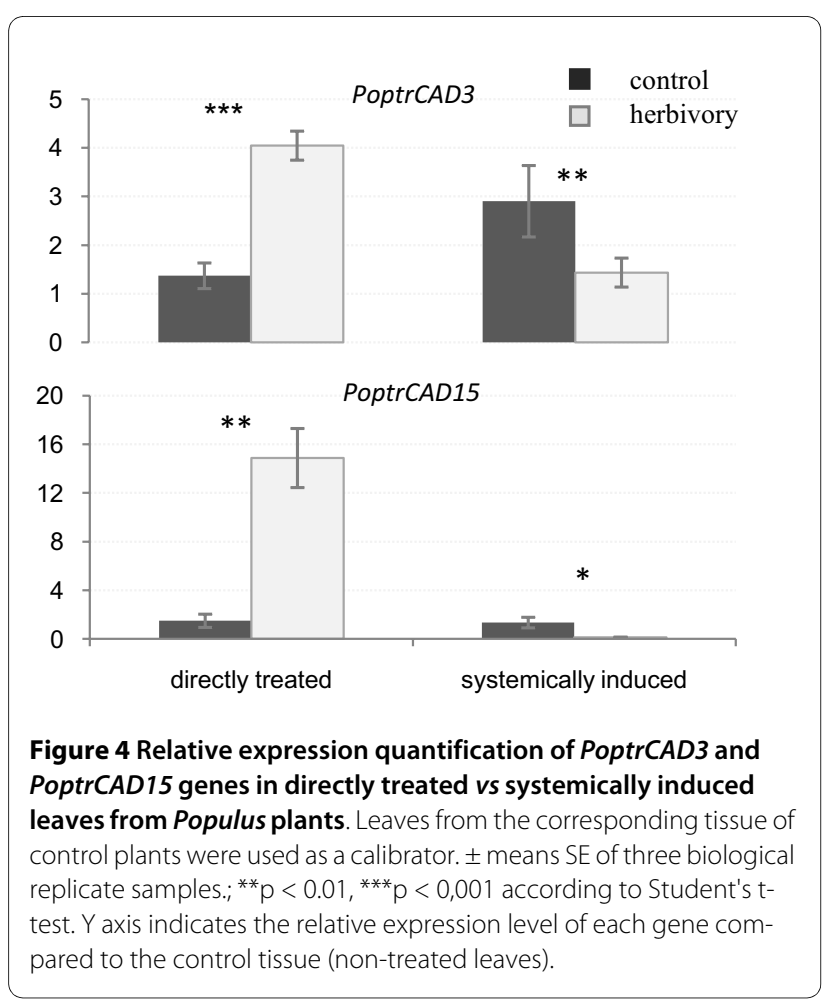

of the current incomplete coverage of the transcriptome of gymnosperm plants. The distribution of bona fide $C A D$ genes (Class I) in a monophyletic clade including sequences from mosses, lycophytes, gymnosperms, and angiosperms is an indication that the real $C A D$ genes existed in the ancestor of land plants and their evolution is intimately associated with the evolution of lignin. This is in agreement with previous studies showing that Selaginella has real lignin [43]. Other studies have reported lignin in red alga Calliarthron cheilosporioides [44], but it was suggested that this was the result of convergent evolution. Class II and Class III corresponding CAD-like genes seems to be the result of duplications that had happen in the ancestor of land plants. Some of these genes still function in lignin biosynthesis under normal growth conditions as was exemplified by PoptrCAD10 and

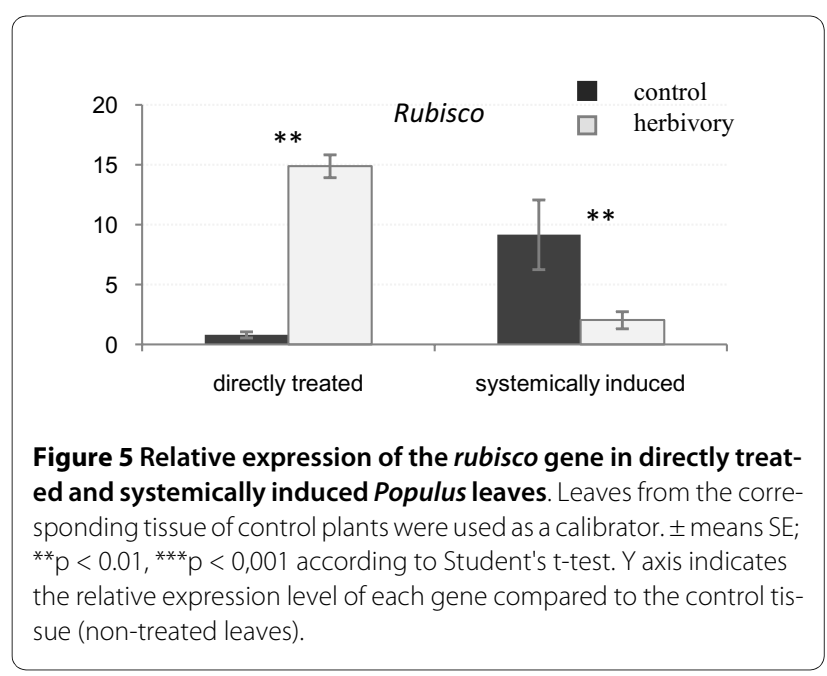


AtCAD6 [33,35]. The other CAD-like genes may function in lignin biosynthesis under various stress conditions or have evolved different functions. For instance, a compilation of Populus gene expression data from the PopGenIE database $[19,45,46]$ showed that several CAD/CAD-like genes (PoptrCAD5, PoptrCAD7, PoptrCAD14, and PoptrCAD12), as well as others not studied here (PoptrCAD6, PoptrCAD13, and PoptrCAD1), were found to be induced under biotic (rust infection) and abiotic stresses (mechanical damage, drought, ozone, UV-B, and elevated $\mathrm{CO} 2)$. Furthermore, it was suggested that the Quercus QsCAD1 gene functions in plant defense against Phytophthora cinnamomi by deactivation of toxins produced by this pathogen [32]. Whatever the nature of defense mechanisms they belong to, the phylogenetic distribution of CAD-like genes suggests that the evolution of these mechanisms had occurred in the ancestor of land plants, which seems to be already targets of pests and pathogens back at that time.

A recent study [33] showed that within the $15 C A D /$ CAD-like genes from Populus trichocarpa, only PoptrCAD4 and PoptrCAD10 genes are preferentially expressed in xylem and seem to be associated with wood development. The other $C A D / C A D$-like genes present various expression profiles and most are induced in leaves or bark. It has been suggested that these genes are involved in defense against plant pathogen attack. For instance, plant treatments with [sulphinyl] acetic acid, 1.1 dimethyl ester (OH-PAS), a specific CAD inhibitor, reduced the penetration resistance of barley to Puccinia hordei. It has been also suggested that some $C A D / C A D$ like genes such as QsCAD1 from Quercus suber are potentially involved in deactivation of toxins produced by phytopathogens [32]. Other studies [47-51] reported a correlation between plant infections by pathogens or herbivore attack and either the amount of lignin or an increase in the expression of lignin pathway associated genes including $C A D / C A D$-like genes. In this study, we analyzed the expression of 10 of the $15 C A D / C A D$-like genes in various tissues of Populus plants challenged with Lymantria dispar and that $C A D / C A D$-like genes present various expression profiles indicating a highly probable functional divergence of genes within this family. Six of the genes studied showed preferential expression in at least one tissue from the stressed plants, suggesting that $C A D / C A D$-like genes act together in defending the plant against pathogen attack. Genes from functional group 1 (PoptrCAD4, PoptrCAD11) and group 3 (PoptrCAD7) showed differential expression in xylem and bark, respectively. PoptrCAD4 and PoptrCAD11 share promoter motifs involved in response to methyl-jasmonate (MeJa), abscisic acid (ABA), and wounding [33]. PoptrCAD7 possess several motifs including the ones involved in MeJa and wound response [33]. This gene was previously reported as having been induced following MeJa, wounding, rust infection, and UV treatments $[19,45,46]$. The induction of those genes in the bark and xylem could be linked to the extensive damage caused to the leaves by the herbivore. It is highly probable that after losing a large fraction of their leaves, the plants respond by protecting the main stem. Genes from group 2 (PoptrCAD3, PoptrCAD4 and PoptrCAD15) showed preferential expression in leaves from herbivore-stressed plants. PoptrCAD4 also showed preferential expression in xylem in stressed plants. Promoter sequence analysis, showed that these three genes shared several motifs including some involved in response to defense against biotic and abiotic stress such as MeJA and ABA [52-55]. This is also in accordance with results from previous studies $[19,45,46]$ showing that poptrCAD4 was induced under MeJA, mechanical damage, drought, UV-B, ozone treatments and rust infection. PoptrCAD15 was shown to be induced under drought and elevated $\mathrm{CO} 2$. The constitutive expression of those genes in leaves under normal conditions and their increase in expression in damaged leaves and stems suggests that these genes play an important role in defense against herbivore attack, or at the very least are responsive to herbivore stress.

PoptrCAD2, PoptrCAD5, PoptrCAD12 and PoptrCAD14 did not show any difference in expression between tissues from stressed and non-stressed plants. These genes present similar motifs as the ones preferentially expressed in tissues from stressed plants. These motifs are involved in response to MeJA, ABA, wounding, and fungal elicitors. The similar expression profile in various tissues between non-stressed and stressed tissues despite the presence in their promoter sequences of several motifs involved in response to biotic and abiotic stresses indicates that those genes may be involved in response to other types of stress than herbivory. This assumption is in accordance with previous results showing that PoptrCAD12 and PoptrCAD14 were induced following MeJA treatment, drought, and elevated $\mathrm{CO} 2$ $[45,46]$.

Three of the ten genes (PoptrCAD3, PoptrCAD11 and PoptrCAD15) analyzed were induced in herbivorestressed plants (Table 1). PoptrCAD3 and PoptrCAD15 genes, which showed similar expression among tissues in the non-stressed plants, were induced in leaves of treated plants. A similar situation was shown for PoptrCAD11, which was induced in the xylem of treated plants. Two genes (PoptrCAD4 and PoptrCAD7) showed a shift in expression profiles between non-stressed and herbivore stressed plants (Table 1). PoptrCAD4, which showed preferential expression in xylem in non-stressed plants, was induced upon herbivory in both xylem and leaves. PoptrCAD7, which was preferentially expressed in leaves in non-stressed plants, showed a high level of expression 
Table 1: List CAD/CAD-like genes induced or suppressed in gypsy moth stressed plants.

\begin{tabular}{|c|c|c|c|c|c|c|}
\hline \multirow[t]{2}{*}{ Gene } & \multicolumn{3}{|c|}{ Normal Growth } & \multicolumn{3}{|c|}{ Stressed } \\
\hline & Leaf & Bark & Xylem & Leaf & Bark & Xylem \\
\hline PoptrCAD4 & & & I & & $\mathrm{SI}$ & 1 \\
\hline PoptrCAD11 & & & & & & $\mathrm{SI}$ \\
\hline PoptrCAD2 & & & 1 & & & 1 \\
\hline PoptrCAD10 & & & I & & & $S R$ \\
\hline PoptrCAD3 & & & & SI & & \\
\hline PoptrCAD15 & & & & SI & & \\
\hline PoptrCAD7 & I & & & & $\mathrm{SI}$ & \\
\hline
\end{tabular}

in the bark of treated plants. These results show that plants induce upon stress the expression of CAD-like genes that are expressed at only low levels during normal plant development. The results also suggest that the expression of other genes such as bona fide lignin biosynthesis genes may shift expression (change specificity) to other tissues to defend against pathogens. These results rule against the hypothesis of absolute functional divergence between bona fide CAD genes and CAD-like genes. It is likely that genes from the three classes are still involved in lignin biosynthesis but have evolved specialized expression profiles.

Phylogenetic analyses of Populus CAD/CAD-like genes showed that PoptrCAD2 and PoptrCAD11 are paralogs. In this study we observed that these two genes present a striking difference in their expression profiles in stressed plants (Table 1). PoptrCAD11 is highly induced in xylem from stressed plants compared to PoptrCAD2. In nonstressed plants, however, both genes showed similar expression patterns in the tissues analyzed [33]. A similar situation was observed for the paralogs PoptrCAD3 and PoptrCAD5. While PoptrCAD5 presented similar expression profiles between non-stressed and stressed plants, PoptrCAD3 was increased in expression in leaves of stressed plants. The expression profiles suggest that the duplication which created the PoptrCAD3 and PoptrCAD5, and the PoptrCAD2 and PoptrCAD11 gene pairs has been followed by a diversification in expression.

Comparison of gene expression in damaged and systemic leaves showed a down regulation of most CAD/ $C A D$-like genes in systemic leaves from stressed plants compared to their counterparts from non-stressed plants. This phenomenon may be linked to a re-allocation of plant resources that has been previously reported, in which the increase in resource utilization for the defense of damaged leaves translates into a slower general metabolism in non-damaged parts of the plant [36,37]. Our result of differential expression of the rubisco gene, as a marker for primary metabolism, in non-treated leaves both in stressed and non-stressed plants, is suggestive that such reallocation of resources is occurring in poplar in response to herbivory, which could help explain the suppressed CAD expression levels systemically in stressed plants. This also indicates that future studies should include the collection of metabolic data to evaluate resource reallocation to confirm if the low expression of $C A D / C A D$-like genes in non-treated tissues reflects a redirection of metabolic flux to the damaged tissues rather than systemic induction of defense genes, rather than a lack of a systemic defense response.

\section{Conclusions}

In conclusion, we confirmed the distribution of $C A D /$ $C A D$-like genes in plants into into three phylogenetic classes that evolved in the ancestor of land plants. We have shown that the expression of genes in the CAD/ $C A D$-like family is coordinated in tissues under both normal growth and under biotic stress conditions. Some CAD/CAD-like genes in Populus that do not function in lignin biosynthesis in xylem may function together in defense against pest attack. We demonstrated that Populus $C A D / C A D$-like genes may play their role in defense in 
a complex tissue-specific manner. We showed that duplicate $C A D / C A D$-like genes have evolved different expression profiles and may have or be evolving towards modified functions as well. Finally, these results take us a step closer to understanding functional divergence among $C A D / C A D$-like genes, a key gene family in the evolution of land plants.

\section{Methods}

\section{Plant material}

Leaves, bark, and stem secondary xylem were collected from young hybrid Populus OGY (P. deltoides $\times$ P. nigra) trees grown in a culture chamber at $25^{\circ} \mathrm{C}$ and $18^{\circ} \mathrm{C}$ during the day and night, respectively. The plants were grown at $16 \mathrm{~h} / 8 \mathrm{~h}$ day/night light regime and at $60 \%$ humidity. For herbivore treatments, we enclosed 10 gypsy moth (Lymantria dispar) larvae within mesh bags that contained leaves from LPI 8-15 (Fig. 6). Control plants were bagged on the same position but without insect larvae. Tissues were harvested from directly treated damaged and systemic (non-damaged three top rows of leaves) after 24 hours herbivory. Tissues were frozen in liquid nitrogen and stored at $-80^{\circ} \mathrm{C}$ until use.

\section{Sequences alignment and phylogenetic analyses}

Sequences used in the phylogenetic analysis include the dataset from [33] to which we added sequences from the genomes of Physcomitrella patens and Selaginella moellendorffii retrieved from JGI [56]. Sequence alignments and phylogenies were constructed as previously described [33].

\section{RNA isolation and CDNA synthesis}

Total RNA was isolated using a CTAB method [57] with minor modifications. The RNA quality and concentration
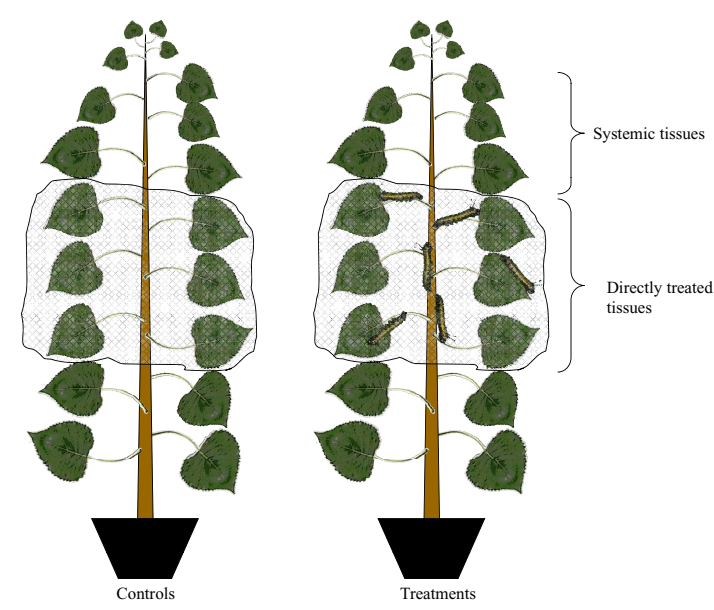

Figure 6 Treatment scheme for the placement of Lymantria dispar larvae on Populus plants. was assessed using an Agilent 2100 Bioanalyzer (Agilent Technologies). To remove any contaminating genomic DNA, RNA samples were treated with RNase-free DNase (Applied Biosystems) before RT-PCR experiments were conducted. RNA was reverse transcribed using random primers from the High Capacity cDNA Reverse Transcription kit (Applied Biosystems) following the manufacturer's recommendations. One microgram of total RNA from each sample was reverse-transcribed for each single RT-PCR reaction.

\section{CAD/CAD-like gene expression analysis using quantitative real time RT-PCR}

Quantitative real time PCR reactions were performed as described previously [33]. In summary, reactions were prepared using the SYBR Green Master Mix kit (Applied Biosystems) and performed in an Applied Biosystems 7500 Fast Real-Time PCR system (Applied Biosystems) with default parameters. Primers used in this study were designed using Primer Express software (Applied Biosystems). We used the gene encoding the $18 \mathrm{~S}$ rRNA as an endogenous control for normalization for template quantity. Dissociation curves were used to verify the specificity of PCR amplification. Expression analyses were performed on three biological replicates (three trees) and three experimental replicates. Data was evaluated using the 7500 Fast System SDS software procedures (Applied Biosystems). Statistical analyses were performed using Statistica 6.0 software (StatSoft Poland Inc., Tulsa, OH, USA).

\section{List of abbreviations used}

CAD: Cinnamyl alcohol dehydrogenase; SAD: Sinapyl Alcohol Dehydrogenase; nt: nucleotide; AA: amino acid; RT-PCR: Reverse transcriptase polymerase chain reaction.

\section{Additional material}

Additional file 1 List of plant genes used in CAD gene phylogenetic analyses. The gene names used in this study, the accession number, species, the database source, and names of previously published genes are indicated. VVGDB, Vignus vinifera genome database; MTGSP, Medicago truncatula genome sequencing project.

Authors' contributions

$A B$ planned the project; designed the experiments, ran the phylogenetic analysis, contributed to the RT-PCR experiments, supervised the work of ABZ and wrote the manuscript. ABZ conducted the expression analyses. CF set up the herbivore stress experiment and assisted in revising the manuscript. JEC provided the financial support, contributed to the discussion of the research plans and results and helped in editing the manuscript. All authors read and approved the final manuscript.

\section{Acknowledgements}

We would like to thank our colleague Scott Diloreto for helping with RNA preparation and for editing an earlier version of the manuscript. We would like to thank Alex Choi for his help on preparing the figures and the tables. Many thanks to Dr. Dawn Luthe for providing access to the Quantitative RT-PCR 
machine. This work was supported by The Schatz Center for Tree Molecular Genetics at Penn State University.

\section{Author Details}

'The School of Forest Resources, and The Huck Institutes of the Life Sciences, Pennsylvania State University, 324 Forest Resources Building, University Park, PA 16802, USA, 2Department of General Botany, Institute of Experimental Biology, Adam Mickiewicz University, Umultowska 89, 61-614 Poznań, Poland and ${ }^{3}$ Center for Chemical Ecology, Pennsylvania State University, University Park, PA 16802, USA

Received: 5 January 2010 Accepted: 28 May 2010

Published: 28 May 2010

\section{References}

1. Sederoff RR, Mackay JJ, Ralph J, Hatfield RD: Unexpected variation in lignin. Curr Opin Plant Biol 1999, 2(2):145-152.

2. Donaldson L, Hague J, Snell R: Lignin Distribution in Coppice Poplar, Linseed and Wheat Straw. Holzforschung 2001, 55(4):379-385.

3. Jourdes M, Cardenas CL, Laskar DD, Moinuddin SG, Davin LB, Lewis NG Plant cell walls are enfeebled when attempting to preserve native lignin configuration with poly-p-hydroxycinnamaldehydes: evolutionary implications. Phytochemistry 2007, 68(14):1932-1956.

4. MacKay JJ, O'Malley DM, Presnell T, Booker FL, Campbell MM, Whetten RW, Sederoff RR: Inheritance, gene expression, and lignin characterization in a mutant pine deficient in cinnamyl alcohol dehydrogenase. Proc Natl Acad Sci USA 1997, 94(15):8255-8260

5. Guillaumie S, Pichon M, Martinant JP, Bosio M, Goffner D, Barriere Y: Differential expression of phenylpropanoid and related genes in brown-midrib bm1, bm2, bm3, and bm4 young near-isogenic maize plants. Planta 2007, 226(1):235-250.

6. Porter KS, Axtell JD, Lechtenberg VL, Colenbrander VF: Phenotype, Fiber Composition, and in vitro Dry Matter Disappearance of Chemically Induced Brown Midrib (bmr) Mutants of Sorghum. Crop Sci 1978, 18(2):205-208

7. Vermerris W, Thompson KJ, McIntyre LM, Axtell JD: Evidence for an evolutionarily conserved interaction between cell wall biosynthesis and flowering in maize and sorghum. BMC Evol Biol 2002, 2:2.

8. Pedersen JF, Vogel KP, Funnell DL: Impact of reduced lignin on plant fitness. Crop Sci 2005, 45:812-819.

9. Kiedrowski S, Kawalleck P, Hahlbrock K, Somssich IE, Dangl JL: Rapid activation of a novel plant defense gene is strictly dependent on the Arabidopsis RPM1 disease resistance locus. The EMBO Journal 1992, 11(13):4677-4684

10. Lao M, Arencibia AD, Carmona ER, Acevedo R, Rodriguez E, Leon O, Santana I: Differential expression analysis by CDNA-AFLP of Saccharum spp. after inoculation with the host pathogen Sporisorium scitamineum. Plant Cell Rep 2008, 27(6):1103-1111.

11. Ithal N, Recknor J, Nettleton D, Maier T, Baum TJ, Mitchum MG Developmental transcript profiling of cyst nematode feeding cells in soybean roots. Mol Plant Microbe Interact 2007, 20(5):510-525.

12. Zabala G, Zou J, Tuteja J, Gonzalez DO, Clough SJ, Vodkin LO Transcriptome changes in the phenylpropanoid pathway of Glycine max in response to Pseudomonas syringae infection. BMC Plant Biol 2006, 6:26.

13. Beaugrand J, Cronier D, Thiebeau P, Schreiber L, Debeire P, Chabbert B: Structure, chemical composition, and xylanase degradation of external layers isolated from developing wheat grain. J Agric Food Chem 2004, 52(23):7108-7117.

14. Bonello P, Pearce RB, Watt F, Grime GW: An induced papilla response in primary roots of Scots pine challenged in vitro with Cylindrocarpon destructans. Physiol Mol Plant Pathol 1991, 39:213-228.

15. Hudgins JW, Christiansen E, Franceschi VR: Induction of anatomically based defense responses in stems of diverse conifers by methyl jasmonate: a phylogenetic perspective. Tree Physiol 2004, 24(3):251-264

16. Mitchell HJ, Hall JL, Barber MS: Elicitor-Induced Cinnamyl Alcohol Dehydrogenase Activity in Lignifying Wheat (Triticum aestivum L.) Leaves. Plant Physiol 1994, 104(2):551-556.

17. Siegrist J, Jeblick W, Kauss H: Defense Responses in Infected and Elicited Cucumber (Cucumis sativus L.) Hypocotyl Segments Exhibiting Acquired Resistance. Plant Physiol 1994, 105(4):1365-1374.
18. Smit F, Dubery IA: Cell wall reinforcement in cotton hypocotyls in response to a Verticillium dahliae elicitor. Phytochemistry 1997, 44:811-815

19. Rinaldi C, Kohler A, Frey P, Duchaussoy F, Ningre N, Couloux A, Wincker P, Le Thiec D, Fluch S, Martin F, et al:: Transcript profiling of poplar leaves upon infection with compatible and incompatible strains of the foliar rust Melampsora larici-populina. Plant Physiol 2007, 144(1):347-366.

20. Franceschi VR, Krokene P, Krekling T, Christiansen E: Phloem parenchyma cells are involved in local and distant defense responses to fungal inoculation or bark-beetle attack in Norway spruce (Pinaceae). Am J Bot 2000, 87(3):314-326

21. Nagy NE, Franceschi VR, Solheim H, Krekling T, Christiansen E: Woundinduced traumatic resin duct development in stems of Norway spruce (Pinaceae): anatomy and cytochemical traits. Am J Bot 2000, 87(3):302-313.

22. Johnson MT, Smith SD, Rausher MD: Plant sex and the evolution of plant defenses against herbivores. Proc Natl Acad Sci USA 2009, 106(43):18079-18084

23. Frost $C J$, Hunter MD: Herbivore-induced shifts in carbon and nitrogen allocation in red oak seedlings. New Phytol 2008, 178(4):835-845.

24. Brodeur-Campbell SE, Vucetich JA, Richter DL, Waite TA, Rosemier JN, Tsai $\mathrm{CJ}$ : Insect herbivory on low-lignin transgenic aspen. Environmental Entomology 2006, 35:1696-1701

25. Chapman SK, Schweitzer JA, Whitham TG: Herbivory differentially alters plant litter dynamics of evergreen and deciduous trees. Oikos 2006 114:566-574.

26. Chapman SK, Hart SC, Cobb NS, Whitham TG, Koch GW: Insect herbivory increases litter quality and decomposition: An extension of the acceleration hypothesis. Ecology 2003, 84:2867-2876.

27. Coley PD, Barone JA: Herbivory and plant defenses in tropical forests. Annual Review of Ecology and Systematics 1996, 27:305-335.

28. Frost CJ, Hunter MD: Insect canopy herbivory and frass deposition affect soil nutrient dynamics and export in oak mesocosms. Ecology 2004, 85:3335-3347.

29. Kurokawa H, Nakashizuka T: Leaf herbivory and decomposability in a Malaysian tropical rain forest. Ecology 2008, 89:2645-2656.

30. Bhuiyan NH, Selvaraj G, Wei Y, King J: Gene expression profiling and silencing reveal that monolignol biosynthesis plays a critical role in penetration defence in wheat against powdery mildew invasion. J Exp Bot 2009, 60(2):509-521.

31. Logemann E, Reinold S, Somssich IE, Hahlbrock K: A novel type of pathogen defense-related cinnamyl alcohol dehydrogenase. Biol Chem 1997, 378(8):909-913.

32. Coelho AC, Horta M, Neves D, Cravador A: Involvement of a cinnamyl alcohol dehydrogenase of Quercus suber in the defence response to infection by Phyutophthora cinnamomi. Phisiological and Molecular Plant Pathology 2006, 69:62-72.

33. Barakat A, Bagniewska-Zadworna A, Plakkat U, Choi A, DiLoreto D, Carlson $\mathrm{J}$ : the Cinnamyl Alcohol Dehydrogenase genes family in Populus: Genome organization, phylogeny, and expression. BMC Plant Biol 2009, 9:26.

34. Li L, Cheng XF, Leshkevich J, Umezawa T, Harding SA, Chiang VL: The last step of syringyl monolignol biosynthesis in angiosperms is regulated by a novel gene encoding sinapyl alcohol dehydrogenase. Plant Cell 2001, 13(7):1567-1586

35. Kim SJ, Kim KW, Cho MH, Franceschi VR, Davin LB, Lewis NG: Expression of cinnamyl alcohol dehydrogenases and their putative homologues during Arabidopsis thaliana growth and development: lessons for database annotations? Phytochemistry 2007, 68(14):1957-1974.

36. Smith AM, Stitt M: Coordination of carbon supply and plant growth. Plant Cell Environ 2007, 30(9):1126-1149.

37. Schwachtje J, Baldwin I: Why does herbivore attack reconfigure primary metabolism? Plant Physiol 2008, 146:845-851.

38. Voelckel C, Weisser WW, Baldwin IT: An analysis of plant-aphid interactions by different microarray hybridization strategies. Mol Ecol 2004, 13(10):3187-3195.

39. Schroder R, Forstreuter M, Hilker M: A plant notices insect egg deposition and changes its rate of photosynthesis. Plant Physiol 2005, 138(1):470-477.

40. Major IT, Constabel CP: Molecular analysis of poplar defense against herbivory: comparison of wound-and insect elicitor-induced gene expression. New Phytol 2006, 172(4):617-635. 
41. Zhu-Salzman K, Salzman RA, Ahn JE, Koiwa H: Transcriptional regulation of sorghum defense determinants against a phloem-feeding aphid. Plant Physio/ 2004, 134(1):420-431.

42. Halitschke R, Gase K, Hui D, Schmidt DD, Baldwin IT: Molecular interactions between the specialist herbivore Manduca sexta (lepidoptera, sphingidae) and its natural host Nicotiana attenuata. VI. Microarray analysis reveals that most herbivore-specific transcriptional changes are mediated by fatty acid-amino acid conjugates. Plant Physiol 2003, 131(4):1894-1902.

43. Weng JK, Li X, Stout J, Chapple C: Independent origins of syringyl lignin in vascular plants. Proc Natl Acad Sci USA 2008, 105(22):7887-7892.

44. Martone PT, Estevez JM, Lu F, Ruel K, Denny MW, Somerville C, Ralph J: Discovery of lignin in seaweed reveals convergent evolution of cellwall architecture. Curr Biol 2009, 19(2):169-175.

45. Sjodin A, Street NR, Sandberg G, Gustafsson P, Jansson S: The Populus Genome Integrative Explorer (PopGenIE): a new resource for exploring the Populus genome. New Phytol 2009.

46. Sjodin A, Bylesjo M, Skogstrom O, Eriksson D, Nilsson P, Ryden P, Jansson S, Karlsson J: UPSC-BASE--Populus transcriptomics online. Plant J 2006, 48(5):806-817.

47. Frost CJ, Mescher MC, Carlson JE, De Moraes CM: Plant defense priming against herbivores: getting ready for a different battle. Plant Physiol 2008, 146(3):818-824.

48. Sibout R, Eudes A, Mouille G, Pollet B, Lapierre C, Jouanin L, Seguin A: CINNAMYL ALCOHOL DEHYDROGENASE-C and -D are the primary genes involved in lignin biosynthesis in the floral stem of Arabidopsis. Plant Cell 2005, 17(7):2059-2076.

49. Kawasaki T, Koita H, Nakatsubo T, Hasegawa K, Wakabayashi K, Takahashi $\mathrm{H}$, Umemura K, Umezawa T, Shimamoto K: Cinnamoyl-CoA reductase, a key enzyme in lignin biosynthesis, is an effector of small GTPase Rac in defense signaling in rice. Proc Natl Acad Sci USA 2006, 103(1):230-235.

50. Kawalleck P, Plesch G, Hahlbrock K, Somssich IE: Induction by fungal elicitor of S-adenosyl-L-methionine synthetase and S-adenosyl-Lhomocysteine hydrolase mRNAs in cultured cells and leaves of Petroselinum crispum. Proc Natl Acad Sci USA 1992, 89(10):4713-4717.

51. Wang G, Ding X, Yuan M, Qiu D, Li X, Xu C, Wang S: Dual function of rice OsDR8 gene in disease resistance and thiamine accumulation. Plant Mol Biol 2006, 60(3):437-449.

52. Xu Y, Chang P, Liu D, Narasimhan ML, Raghothama KG, Hasegawa PM, Bressan RA: Plant Defense Genes Are Synergistically Induced by Ethylene and Methyl Jasmonate. Plant Cell 1994, 6(8):1077-1085

53. Sang JR, Jiang MY, Lin F, Li J, Xu SC: Role of nitric oxide in abscisic acidinduced subcellular antioxidant defense of maize leaves. Zhi Wu Sheng Li Yu Fen Zi Sheng Wu Xue Xue Bao 2007, 33(6):553-566.

54. Anderson JP, Badruzsaufari E, Schenk PM, Manners JM, Desmond OJ, Ehlert C, Maclean DJ, Ebert PR, Kazan K: Antagonistic interaction between abscisic acid and jasmonate-ethylene signaling pathways modulates defense gene expression and disease resistance in Arabidopsis. Plant Cell 2004, 16(12):3460-3479.

55. Zhang A, Jiang M, Zhang J, Ding H, Xu S, Hu X, Tan M: Nitric oxide induced by hydrogen peroxide mediates abscisic acid-induced activation of the mitogen-activated protein kinase cascade involved in antioxidant defense in maize leaves. New Phyto/ 2007, 175(1):36-50.

56. Joint Genome Institute: [http://www.jgi.doe.gov]

57. Chang S, Puryear J, Cairney J: A simple and efficient method for isolating RNA from pine trees. Plant Molecular Biology Reporter 1993, 11:113-116.

\section{Submit your next manuscript to BioMed Central} and take full advantage of:

- Convenient online submission

- Thorough peer review

- No space constraints or color figure charges

- Immediate publication on acceptance

- Inclusion in PubMed, CAS, Scopus and Google Scholar

- Research which is freely available for redistribution

Submit your manuscript at www.biomedcentral.com/submit
C Biomed Central 